ARTICLE

Received 7 Sep 2016 | Accepted 8 Feb 2017 | Published 28 Mar $2017 \quad$ DOl: 10.1038/ncomms14876 $\quad$ OPEN

\title{
A redox-mediated Kemp eliminase
}

\author{
Aitao Li ${ }^{1,2, \star}$, Binju Wang ${ }^{3, \star}$, Adriana Ilie ${ }^{1,2}$, Kshatresh D. Dubey ${ }^{3}$, Gert Bange ${ }^{4}$, Ivan V. Korendovych ${ }^{5}$, \\ Sason Shaik ${ }^{3} \&$ Manfred T. Reetz ${ }^{1,2}$
}

The acid/base-catalysed Kemp elimination of 5-nitro-benzisoxazole forming 2-cyano-4nitrophenol has long served as a design platform of enzymes with non-natural reactions, providing new mechanistic insights in protein science. Here we describe an alternative concept based on redox catalysis by P450-BM3, leading to the same Kemp product via a fundamentally different mechanism. QM/MM computations show that it involves coordination of the substrate's $\mathrm{N}$-atom to haem-Fe(II) with electron transfer and concomitant $\mathrm{N}-\mathrm{O}$ heterolysis liberating an intermediate having a nitrogen radical moiety Fe(III)-N• and a phenoxyl anion. Product formation occurs by bond rotation and $\mathrm{H}$-transfer. Two rationally chosen point mutations cause a notable increase in activity. The results shed light on the prevailing mechanistic uncertainties in human P450-catalysed metabolism of the immunomodulatory drug leflunomide, which likewise undergoes redox-mediated Kemp elimination by P450-BM3. Other isoxazole-based pharmaceuticals are probably also metabolized by a redox mechanism. Our work provides a basis for designing future artificial enzymes.

\footnotetext{
${ }^{1}$ Department of Biocatalysis, Max-Planck-Institut für Kohlenforschung, Kaiser-Wilhelm-Platz 1, Mülheim an der Ruhr 45470, Germany. ${ }^{2}$ Department of Chemistry, Philipps-Universität Marburg, Marburg 35032, Germany. ${ }^{3}$ Institute of Chemistry and the Lise Meitner-Minerva Center for Computational Quantum Chemistry, The Hebrew University of Jerusalem, Jerusalem 9190401, Israel. ${ }^{4}$ LOEWE Center for Synthetic Microbiology (SYNMIKRO) and Department of Chemistry, Philipps-Universität Marburg, Marburg 35032, Germany. ${ }^{5}$ Department of Chemistry, Syracuse University, 111 College Place, Syracuse, New York 13244, USA. * These authors contributed equally to this work. Correspondence and requests for materials should be addressed to S.S. (email: sason.shaik@gmail.com) or to M.T.R. (email: reetz@mpi-muelheim.mpg.de).
} 
T he Kemp elimination ${ }^{1}$ of 5 -nitrobenzisoxazole has become a de facto experimental springboard for designing new protein catalysts to uncover the principles that govern enzymatic catalysis. For more than two decades ${ }^{2}, \mathrm{Kemp}$ eliminases based on various protein scaffolds have provided valuable insights into understanding and mimicking enzymes ${ }^{2-15}$ (Fig. 1a). Combining computational design methodology with 17 rounds of state-of-the-art directed evolution Hilvert, Mayo and coworkers reported the artificial enzyme HG3.17, which employs a Brønsted acid/base mechanism to catalyse Kemp elimination with an unprecedented catalytic efficiency that approaches the activities of natural enzyme $\left(k_{\mathrm{cat}}=700 \mathrm{~s}^{-1}\right.$ and $\left.k_{\text {cat }} / K_{\mathrm{m}}=230,000 \mathrm{M}^{-1} \mathrm{~s}^{-1}\right)^{16}$. While these numbers are truly impressive, analysis of the possible efficiency limits for base-catalysed Kemp elimination shows that additional improvement is still possible in principle ${ }^{17}$. Along a different line, it has been postulated that an oxidoreductase breaks down 5-nitrobenzisoxazole possibly by an oxidative process, although no mechanistic evidence was reported ${ }^{18}$.

In our work, we wanted to identify a catalyst that employs a mechanism beyond acid-base mechanism, specifically a redox process. From a fundamental point, finding a protein scaffold that catalyses the Kemp elimination by a fundamentally different mechanism would not only further our understanding of the function, genesis and evolution of enzymes, but also provide new opportunities for creating catalysts for novel chemical transformations.

Since enzymes that facilitate redox reactions are quite abundant, we speculated that a redox-based mechanism should be possible in Kemp elimination. We focused on cytochrome P450 monooxygenases (CYPs) for several reasons: Human cytochrome P450 (CYP) enzymes play a crucial role in the metabolism of therapeutic drugs ${ }^{19-21}$, including the degradation of a number of prominent isoxazole-based pharmaceuticals ${ }^{22-24}$. A prime example is the metabolism of leflunomide, an anti-inflammatory agent used in the treatment of rheumatoid arthritis ${ }^{23}$. The main metabolic outcome is the physiologically active teriflunomide (2-cyano-3-oxo- $\mathrm{N}$-[(4trifluormethyl)phenyl]butyramide, also called A771726), a formal Kemp elimination product. Two pathways have been postulated, an acid/base and an undefined redox-mediated process $^{22,23}$, but mechanistic studies to distinguish between the two possibilities have not been reported to date. The term Kemp elimination was not referred to in this connection. CYPs are remarkably promiscuous, lending themselves to non-natural reactions as shown, for example, by the seminal report of Dawson, Breslow and coworkers describing CYP as a catalyst in inter- and intramolecular nitrene $\mathrm{CH}$ insertion ${ }^{25}$ and by the recent discovery of related reactions that can be catalysed by this class of enzymes ${ }^{26}$. Moreover, rat liver microsomes that contain CYPs were reported to catalyse the dehydration of aldoximes, either Lewis acid/Brønsted base catalysis or some kind of a redox process being postulated ${ }^{27}$. Clearly, in these biological transformations mechanistic ambiguities persist to this day.

Here we show on the basis of experimental and computational data that the biocatalytic Kemp elimination of 5-nitro-benzisoxazole with formation of 2-cyano-4-nitrophenol need not proceed by the traditional acid/base mechanism. Cytochrome P450 monooxygenase from Bacillus megaterium (P450-BM3) and rationally designed mutants constitute active Kemp eliminases that indeed follow a redox-mediated mechanism. This finding has ramifications regarding the human metabolism of the immunomodulatory therapeutic drug leflunomide and other isoxazolebased pharmaceuticals.

\section{Results}

Kemp elimination activity test with wild-type P450-BM3. We speculated that the ferrous haem cofactor in the selfsufficient P450-BM3 (refs 21,28) could coordinate to the most basic position of substrate $\mathbf{1}$ at the isoxazole nitrogen. An internal redox process was then expected in which $\mathrm{Fe}(\mathrm{II})$ is oxidized to $\mathrm{Fe}(\mathrm{III})$ by electron-flow to the electron-deficient 5-nitrobenzisoxazole nucleus with concurrent rupture of the weakest bond $(\mathrm{O}-\mathrm{N})$, followed by proton transfer and formation of the formal Kemp elimination product 2 (Fig. 1b).

Wildtype (WT) P450-BM3 rapidly converts substrate 1 into 2 in a cell free extract (CFE) in the presence of NADPH for keeping the haem cofactor reduced. To exclude the possibility that an unknown protein in the CFE may catalyse this Kemp elimination, the enzyme was purified and tested, and the product identity was confirmed by gas chromatography-mass spectrometry (GC-MS) analysis (Supplementary Figs 1 and 2). Kinetic experiments using purified enzyme showed that activity of WT P450-BM3 in the Kemp elimination is remarkable. The observed turnover number $\left(k_{\mathrm{cat}} / K_{\mathrm{m}}=240 \pm 60 \mathrm{~s}^{-1} \mathrm{M}^{-1}\right.$; $k_{\text {cat }}>1.5 \mathrm{~s}^{-1} ; K_{\mathrm{m}}>6 \mathrm{mM}$, exact $k_{\text {cat }}$ and $K_{\mathrm{m}}$ values are only estimated due to low substrate solubility) is higher than those of any of the previously designed catalysts (including catalytic antibodies) before the application of directed evolution, as well as serum albumins ${ }^{4}$.

a

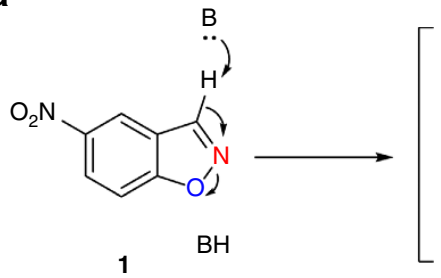

b<smiles></smiles>

1<smiles>[B]Cc1noc2ccc([N+](=O)[O-])cc12</smiles>

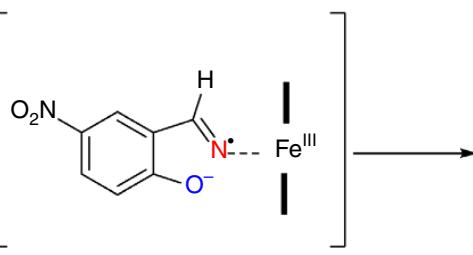

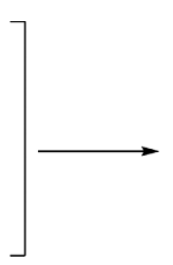<smiles>N#Cc1cc([N+](=O)[O-])ccc1O</smiles>

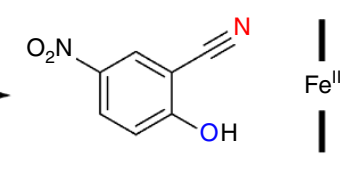


Experimental evidence for the redox mechanism. A number of control experiments were designed to support the mechanistic hypothesis of a redox process. To begin with, experiments done with the purified enzyme showed that in the absence of NADPH no reaction occurs, while the addition of carbon monoxide (CO) to the reaction mixture resulted in a 10 -fold loss of activity (Supplementary Table 1). Moreover, conducting the reaction under anaerobic conditions led to an almost twofold activity improvement (Supplementary Table 2). These findings are consistent with competition of $\mathrm{CO}$ or oxygen with substrate $\mathbf{1}$ in coordinating to ferrous haem. Thus, they are not compatible with a classical acid/base mechanism. On this basis we concluded that haem-Fe(II) is essential for the P450-BM3 catalysed Kemp elimination, and that a redox mechanism is likely. Further strong evidence for a redox mechanism was gained by substituting haem-Fe(II) in WT P450-BM3 with haem-Zn(II) according to a well-tested and reliable procedure ${ }^{29}$ (Supplementary Figs 3 and 4). P450-BM3 containing $\mathrm{Zn}$ (II) failed to catalyse the Kemp elimination of substrate 1, while the activity was fully recovered by the reconstituted protein with Fe-porphyrin IX (hemin) (Supplementary Table 3). Here again, the results are incompatible with an acid/base mechanism.

Evidence for a redox mechanism was also gained by a mutational experiment. It is well known that a cysteine-toserine mutation ${ }^{30}$ in $\mathrm{P} 450-\mathrm{BM} 3$ at the axial Fe-binding position C400S eliminates monooxygenation activity but enhances the efficiency of $\mathrm{Fe}^{\mathrm{III}}$-to- $\mathrm{Fe}^{\mathrm{II}}$ reduction by using $\mathrm{NADPH}$ as a reductant $^{26}$. Therefore, we tested P450-BM3 variant C400S in the Kemp elimination of $\mathbf{1}$. As anticipated on the basis of a redox mechanism, it showed an almost sixfold improvement in the catalytic efficiency $\left(k_{\mathrm{cat}} / K_{\mathrm{m}}\right)$ relative to WT P450-BM3 $\left(k_{\text {cat }} / K_{\mathrm{m}}=1,400 \pm 150 \mathrm{~s}^{-1} \mathrm{M}^{-1} ; k_{\mathrm{cat}}=3.8 \pm 0.4 \mathrm{~s}^{-1} ; K_{\mathrm{m}}=2.7 \pm\right.$ $\left.0.4 \mathrm{mM} ; k_{\text {cat }} / k_{\text {uncat }}=3.3 \times 10^{6}\right)$.

Mechanistic evidence by $\mathrm{MD} /(\mathrm{QM} / \mathrm{MM})$ computations. To elucidate the mechanistic details of the redox-mediated Kemp elimination in WT P450-BM3, we performed molecular dynamic (MD) simulations and quantum mechanics/molecular mechanics (QM/MM) calculations. These computations revealed no evidence for a pose of substrate $\mathbf{1}$ in which a classical acid/base mechanism is possible (Fig. 2). In addition, a $\pi-\pi$ interaction between the phenyl group of phenylalanine (F87) and the substrate was identified, which sterically prevents tight binding of 1 with haem-Fe(II) necessary for optimal redox reaction (Fig. 3a). MD simulations for the oxidized haem state showed that the substrate never approach the ferric haem moiety (Supplementary Fig. 5). As such, the key reactive species is the ferrous and not the ferric haem species.

Based on a well-tested and reliable procedure for metalloenzymes $^{31-33}$, QM/MM calculations were performed by using the equilibrated snapshots from the MD simulations of the $\mathrm{Fe}(\mathrm{II})$ haem state (see Supplementary Methods for details). Figure 3b presents the QM/MM calculated energy profile for the Kemp elimination of 1 in WT P450-BM3. As the MD simulation reveals (Fig. 3a), the substrate has an upright orientation, in which its $\mathrm{N}$-atom is directed towards the Fe(II) center, while the $\mathrm{H}$-atom is pointing down toward the haem. Moreover, no base residue is identified in the vicinity of this $\mathrm{H}$ to accept the substrate proton. Starting from the initial reactant complex of $\mathbf{1}$, the attack of the substrate's $\mathrm{N}$-atom onto $\mathrm{Fe}(\mathrm{II})$ is coupled with electron transfer from the latter, thus leading to a heterolytic cleavage of $\mathrm{N}-\mathrm{O}$ bond. No computational evidence for a short-lived Lewis acid/ Brønsted base complex was evident (Supplementary Fig. 7), which is partially because the haem is reduced (negatively charged), and there is significant electrostatic repulsion between

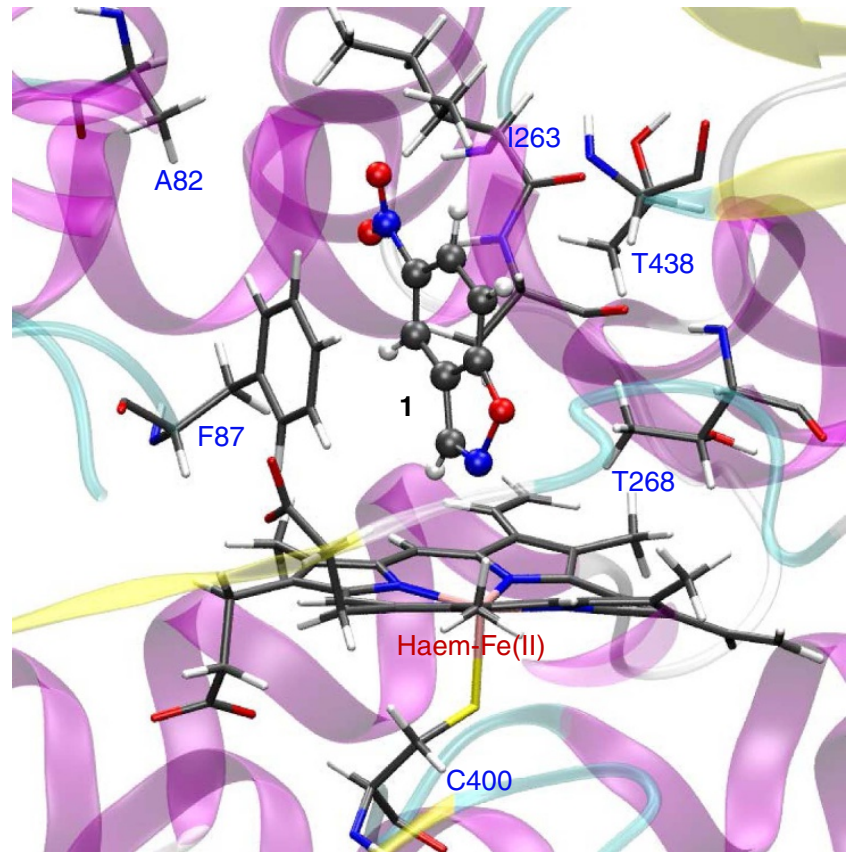

Figure 2 | Overview of substrate 1 accommodated in the active site of WT P450-BM3. The representative snapshot was taken from molecular dynamic (MD) simulation, revealing substrate $\mathbf{1}$ in the binding pocket of P450-BM3 and the surrounding residues. As can be seen, there are no residues which could act as in an acid/base mechanism.

the substrate's $\mathrm{N}$ and the haem moiety. According to the calculations, the electron transfer from $\mathrm{Fe}(\mathrm{II})$ to the substrate generates intermediate IN1 (Fig. 3b), wherein the $\mathrm{N}-\mathrm{O}$ bond is broken, and the haem iron is bonded to the nitrogen to form the nitrogen radical moiety $\mathrm{Fe}(\mathrm{III})-\mathrm{N}^{\bullet}$ and a phenoxyl anion. The spin density at $\mathrm{N}$ is -0.62 , indicating notable spin localization at this atom. Subsequent bond rotation places $\mathrm{H}$ close to $\mathrm{O}$ via TS2 with formation of intermediate IN2 (Fig. 3b). Mulliken charge analysis reveals the transfer of a significant charge $(-0.74 \mathrm{e})$ from $\mathrm{Fe}$ (II) to the substrate in TS2, and as such we are dealing with a redox-mediated mechanism. The final proton transfer to $\mathrm{O}^{-}$via TS3 leads to the final product and regeneration of Fe(II) (Fig. 3b). The reactions involve two-state reactivity ${ }^{34,35}$. The reaction starts with the quintet ground state of $\mathrm{Fe}(\mathrm{II})$, but crosses to the triplet state already at the first step of the reaction. The calculated overall barrier (via ${ }^{3}$ TS2) with zero-point energy correction is $21.1 \mathrm{kcal} \mathrm{mol}^{-1}$.

For comparison, we also investigated the reactivity of $\mathrm{Zn}$ (II)haem with QM/MM calculations. Our QM/MM calculations show the substrate activation via $\mathrm{Zn}$ (II)-haem experiences a huge barrier (over $50 \mathrm{kcal} \mathrm{mol}^{-1}$ in Supplementary Fig. 8). Moreover, no stable intermediate like IN1 could be located. This result is consistent with the experimental findings and redox-mediated process in $\mathrm{Fe}(\mathrm{II})$-haem, since $\mathrm{Zn}$ (II) is usually not a redox center. Finally, we compared the positive change on $\mathrm{Fe}(\mathrm{II})$ in $\mathrm{Fe}(\mathrm{II})$ haem and $\mathrm{Zn}$ (II) in $\mathrm{Zn}(\mathrm{II})$-haem with QM/MM calculations, and found the positive charge on $\mathrm{Zn}(\mathrm{II})(+0.74 \mathrm{e})$ is even bigger than that in $\mathrm{Fe}(\mathrm{II})(+0.66 \mathrm{e})$, this excludes the possibility that $\mathrm{Fe}(\mathrm{II})$ plays a purely electrostatic role in the concerted elimination reaction and further supports the redox-mediated mechanism.

Engineering P450-BM3 for activity improvement. Led by these computational predictions, we turned to mutational studies. Since optimal binding of substrate $\mathbf{1}$ to ferrous haem appeared to be 
a
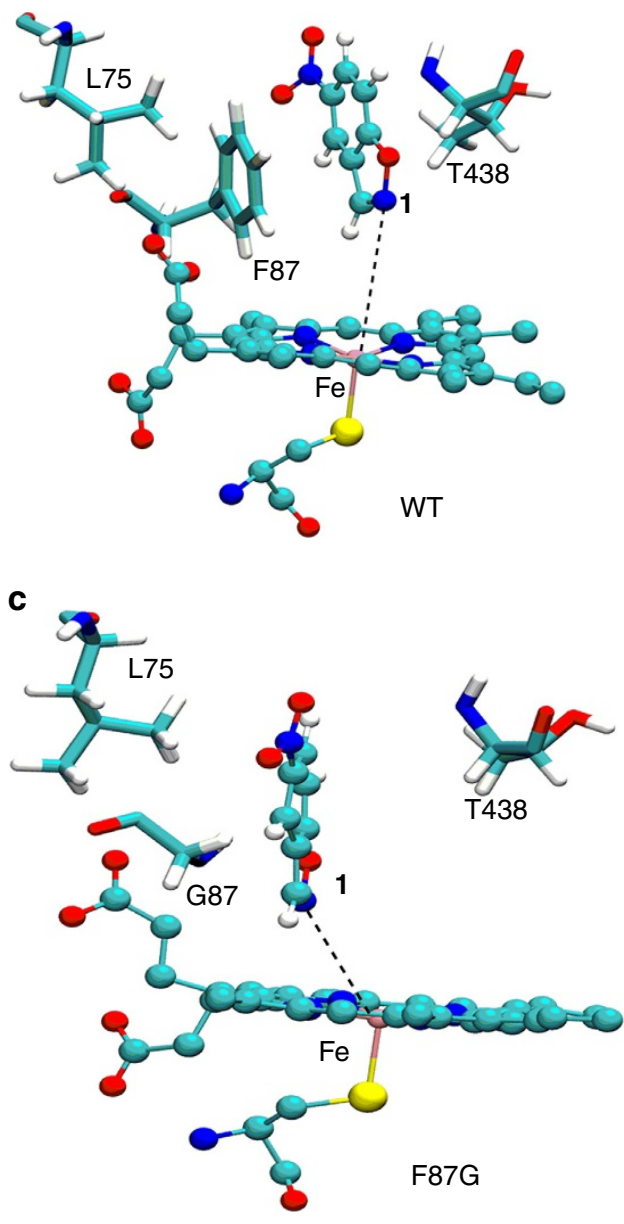

b

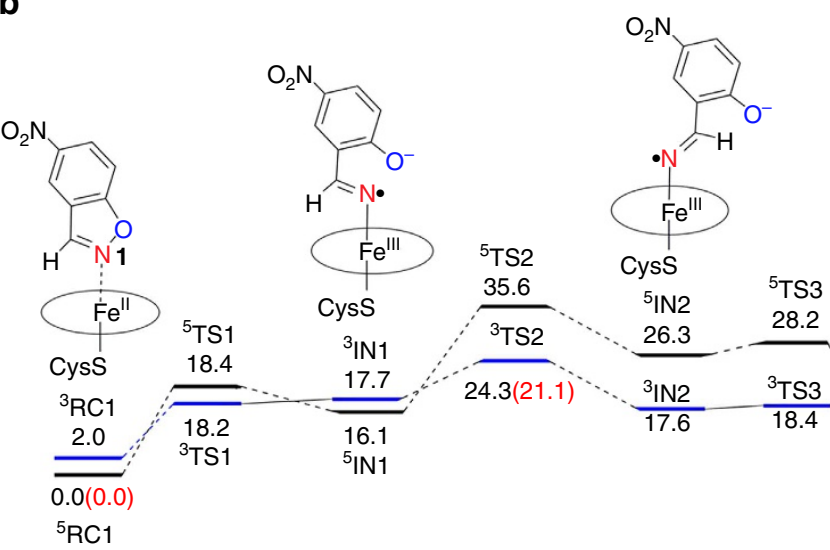

d

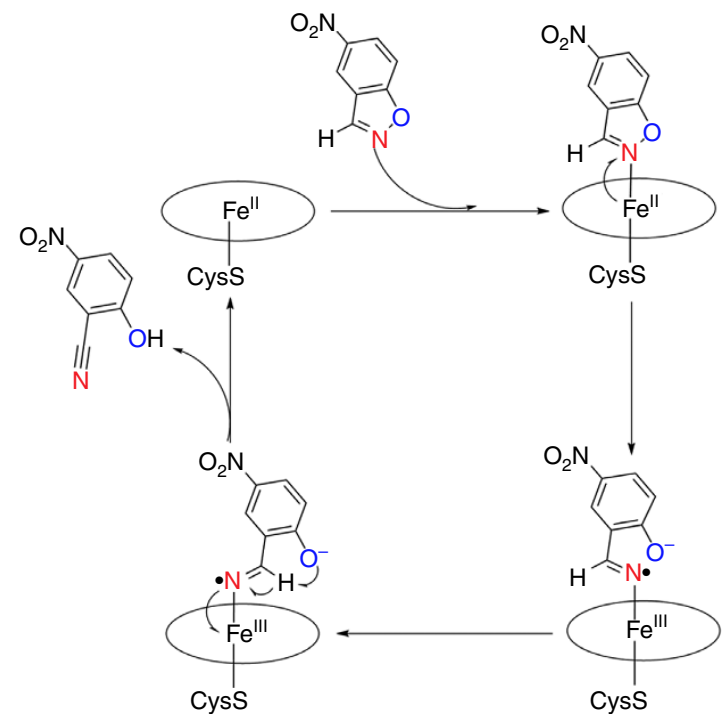

Figure 3 | Redox-mediated Kemp elimination of substrate 1. (a) A representative snapshot in the equilibrium molecular dynamic (MD) trajectory showing the active site structure of WT P450-BM3; note that the nitrogen atom of substrate $\mathbf{1}$ is directed toward Fe(II), with an average Fe-N1 distance of 4.44 $\AA$. (b) Quantum mechanics/molecular mechanics (QM/MM) (UB3LYP/B2) relative energies (kcal mol ${ }^{-1}$ ) for the redox-mediated Kemp elimination of 1. All values are dispersion-corrected. The values in parentheses also include zero-point energy (ZPE) corrections. For clarity, the reactivity energy profile in the singlet state is provided in Supplementary Fig. 6. Cartesian coordinates of QM region for all species from QM/MM calculations are available as Supplementary Data 1. (c) A representative snapshot in the equilibrium MD trajectory showing the active site structure of variant F87G, with an average $\mathrm{Fe}-\mathrm{N} 1$ distance of $4.11 \AA$. (d) Catalytic cycle for redox-mediated Kemp elimination.

Table 1 | Summary of kinetic parameters for P450-BM3 and variants catalysing the Kemp elimination of 1.

\begin{tabular}{lcccc} 
Catalyst $^{\star}$ & $\boldsymbol{K}_{\mathbf{m}}(\mathbf{m M})$ & $\boldsymbol{k}_{\text {cat }}\left(\mathbf{s}^{-\mathbf{1})}\right.$ & $\boldsymbol{k}_{\text {cat }} / \boldsymbol{K}_{\mathbf{m}}\left(\mathbf{s}^{-\mathbf{1}} \mathbf{M}^{-\mathbf{1})}\right.$ & $\boldsymbol{k}_{\text {cat }} / \boldsymbol{K}_{\text {uncat }}^{\dagger}$ \\
\hline WT P450-BM3 & $>6$ & $>1.5$ & $240 \pm 60$ & $1.3 \times 10^{6}$ \\
C400S & $2.7 \pm 0.4$ & $3.8 \pm 0.4$ & $1,400 \pm 150$ & $3.3 \times 10^{6}$ \\
F87G & $2.1 \pm 0.4$ & $2.7 \pm 0.3$ & $1,300 \pm 140$ & $2.3 \times 10^{6}$ \\
F87A & $2.0 \pm 0.3$ & $1.2 \pm 0.1$ & $600 \pm 50$ & $1.0 \times 10^{6}$ \\
F87V & $0.50 \pm 0.04$ & $1.1 \pm 0.1$ & $2,200 \pm 200$ & $0.9 \times 10^{6}$ \\
F87I & $>4$ & $>1.5$ & $320 \pm 40$ & $1.3 \times 10^{6}$ \\
A82F & $0.27 \pm 0.03$ & $8.4 \pm 0.4$ & $31,000 \pm 1,500$ & $7.0 \times 10^{6}$ \\
F87G/A82F & $1.3 \pm 0.2$ & $11.5 \pm 0.7$ & $8,800 \pm 700$ & $10.0 \times 10^{6}$ \\
A82F/C40OS & $0.61 \pm 0.13$ & $2.9 \pm 0.4$ & $4,800 \pm 700$ & $2.5 \times 10^{6}$ \\
\hline
\end{tabular}

*Assay conditions: $25^{\circ} \mathrm{C}, 50 \mathrm{mM}$ sodium phosphate buffer $\mathrm{pH} 8.0,100 \mathrm{mM} \mathrm{NaCl}, 0.25 \mathrm{mM} \mathrm{NADPH}, 100 \mathrm{nM}$ or $300 \mathrm{nM}$ purified enzyme, $5 \%$ acetonitrile. Errors correspond to \pm s.d. determined from at least three independent measurements.

$\dagger$ TRate accelerations for the Kemp eliminases were calculated based on the rate constant for the uncatalyzed reaction determined in ref. $10\left(k_{\text {uncat }}=1.16 \times 10^{-6} \mathrm{~s}^{-1}\right)$.

prevented sterically by phenylalanine at residue F87, we introduced smaller amino acids at this position. As listed in Table 1, mutant F87I shows comparable activity as WT P450-BM3 due to similar size of Ile and Phe. The $K_{\mathrm{m}}$ values for mutants F87V, F87A and F87G are significantly lowered, and the highest activity was observed for F87G. The catalytic efficiency $\left(k_{\text {cat }} / K_{\mathrm{m}}\right)$ of this 


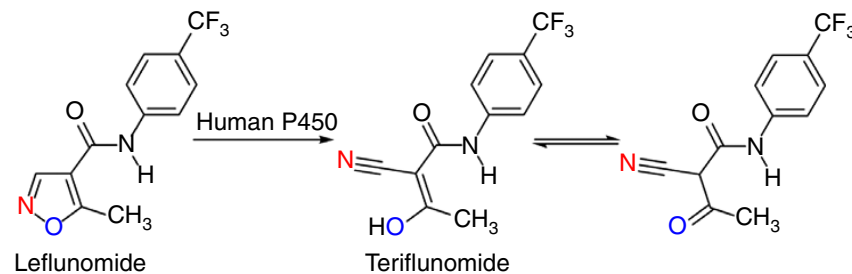

Figure 4 | Metabolism of lefinomide with human P450. Human P450 catalyses the isoxazole ring scission of leflunomide, an immunomodulatory therapeutic drug $22-24$, leading to teriflunomide (A771726).

variant is more than fivefold higher relative to WT P450-BM3 due to the lower Michaelis constant $K_{\mathrm{m}}$ and improved activity $k_{\text {cat }}$ (Table 1). The subsequent MD simulations (Fig. 3c) revealed that the $\mathrm{Fe}(\mathrm{II})-\mathrm{N}_{\text {benzisoxazole }}$ distance in variant $\mathrm{F} 87 \mathrm{G}$ is indeed shorter than in the case of the WT enzyme (see Supplementary Fig. 9 for details).

Next, we attempted to reshape the large substrate binding pocket of P450-BM3 to better match the small size of the substrate. The A82F mutation in P450-BM3 was previously shown to greatly enhance the enzyme's binding affinity for small molecules, leading to significant improvements in catalytic hydroxylation efficiency ${ }^{36}$. Therefore, we introduced the A82F mutation into both WT P450-BM3 and F87G P450-BM3. Kinetic characterization of the two resulting variants showed 129and 36-fold improvements in catalytic efficiencies $\left(k_{\mathrm{cat}} / K_{\mathrm{m}}\right)$ in the $\mathrm{A} 82 \mathrm{~F}$ and $\mathrm{A} 82 \mathrm{~F} / \mathrm{F} 87 \mathrm{G}$ mutants, respectively, due to both improved turnover number and the Michaelis constant (Table 1). These results underscore the predictive power of the combination of $\mathrm{MD}$ and $\mathrm{QM} / \mathrm{MM}$ with empirical observations that allowed the identification of Kemp eliminase activity based on a novel redox-mediated mechanism (Fig. 3d). The turnover number observed for the rationally designed A82F mutant obtained after screening only three additional variants is higher than in any of the previously designed Kemp eliminases with the exception of HG3.17 (ref. 16).

Metabolism of leflunomide by P450-BM3. Following the work with the model compound $\mathbf{1}$, we turned to leflunomide as substrate, knowing that this compound is metabolized by human P450 (refs 22-24) (Fig. 4). As anticipated, WT P450-BM3 as well as selected variants A82F, F87G, C400S and A82F/F87G proved to be active with formation of the formal Kemp elimination product teriflunomide (A771226) (Fig. 5, Supplementary Figs 10 and 11). In full agreement with the results obtained for $\mathbf{1}$, the A82F mutant is $\sim 8$-fold more active in metabolizing leflunomide as compared to WT P450-BM3. With the exception of variant C400S, they all led to a small amount of side-product, likely to be the hydroxylation product as reported earlier in human P450-catalysed metabolism of leflunomide ${ }^{37}$. Based on our experimental and computational results using P450-BM3 as the catalyst in the reaction of the standard substrate $\mathbf{1}$, the same redox mechanism may be postulated in human metabolism of leflunomide, but these mechanistic details still need to be explored. Relevant is also the reported human metabolism of a different isoxazole-based therapeutic drug, zonisamide ${ }^{23}$, active in the treatment of epilepsy, Parkinson's disease, dardive dyskinesia, migraine and obesity. The metabolite is a ring-opened compound, namely 2-(sulfamoylacetyl)phenol (Supplementary Fig. 12), which cannot be formed by a classical acid/base-mediated Kemp elimination since it lacks a hydrogen at the 3-position. In this case a redox mechanism could be involved, but this needs to be checked in future research.

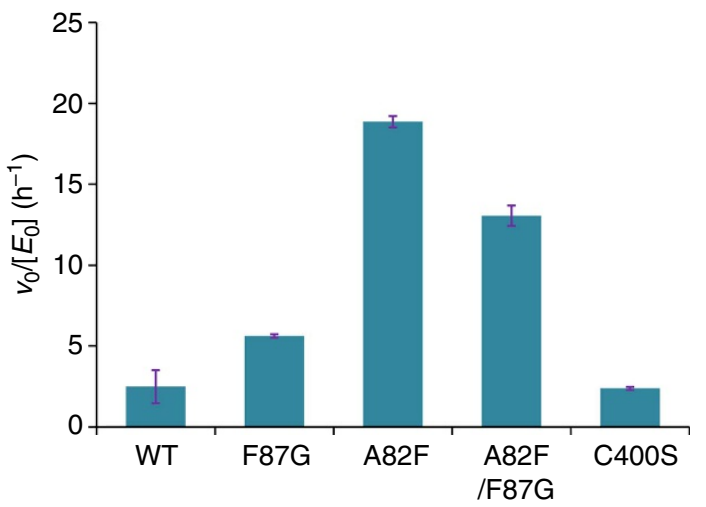

Cytochrome P450-BM3 variants

Figure 5 | Activity test of P450-BM3 variants in metabolizing leflunomide. Reaction conditions: $500 \mu$ reaction consisting of $1 \mu \mathrm{M}$ enzyme, $500 \mu \mathrm{M}$ substrate and $1 \mathrm{mM} \mathrm{NADPH}$ in phosphate buffer $(50 \mathrm{mM}$, $\mathrm{pH} 8.0,100 \mathrm{mM} \mathrm{NaCl}), 25^{\circ} \mathrm{C}, 1,000$ r.p.m. for $1 \mathrm{~h}$. All reactions were performed in triplicate, and error bars show \pm s.d.

\section{Discussion}

Various protein scaffolds have been reported as Kemp eliminases, all operating by the traditional acid/base mechanism ${ }^{2-17}$. In contrast, we have identified a Kemp eliminase that functions by a fundamentally different mechanism based on a redox process. WT P450-BM3 and several rationally designed mutants are capable of efficiently catalysing ring-opening of the standard model compound 5-nitro-benzisoxazole 1 with formation of the formal Kemp product 2-cyano-4-nitrophenol 2. Extensive MD and QM/MM calculations coupled with mechanistic and mutational studies clearly point to a redox-mediated bond cleavage mechanism as opposed to the conventional acid/base process. The experimental evidence is summarized by the following points: in the absence of NADPH no reaction occurs; addition of $\mathrm{CO}$ impedes activity; reaction under anaerobic conditions results in twofold activity improvement; especially mutants A82F, F87G, C400S, A82F/F87G and A82F/C400S lead to improvements in catalytic efficiency; the haem- $\mathrm{Zn}$ (II) analogue of WT P450-BM3 shows no activity in the standard reaction of substrate 1.

WT P450-BM3 catalyses the Kemp elimination via the redox path with an efficiency $\left(k_{\mathrm{cat}} / K_{\mathrm{m}}=240 \pm 60 \mathrm{~s}^{-1} \mathrm{M}^{-1}\right)$ that is higher than those of any previously designed Kemp eliminases in the absence of mutations introduced by directed evolution, as well as serum albumins ${ }^{4}$. Several rationally designed mutants resulted in pronounced rate enhancement, variant A82F showing the best performance $\left(k_{\mathrm{cat}} / K_{\mathrm{m}}=31,000 \pm 1,500 \mathrm{~s}^{-1} \mathrm{M}^{-1}\right)$, which corresponds to a $k_{\text {cat }} / k_{\text {uncat }}$ value of $7.0 \times 10^{6}$. Further improvements can be expected by applying directed evolution, or possibly by modifying the metal centre ${ }^{29}$.

Importantly, we have also shown that WT P450-BM3 and mutants thereof catalyse the transformation of the therapeutic drug leflunomide with formation of the formal Kemp product teriflunomide (A771226) (Figs 4 and 5). This transformation was known to be involved in the metabolism of leflunomide in humans, but its mechanism has remained elusive $^{22}$. We believe that the redox mechanism shown in Fig. 3b will inspire respective research of human P450mediated metabolism of leflunomide and of other isoxazolebased pharmaceuticals such as zonisamide ${ }^{23}$, hopefully ending any remaining mechanistic ambiguities. Finally, a variety of redox-catalysed promiscuous reactions can be envisioned for practical applications, extending the list of $\mathrm{P} 450$-mediated non-natural reactions ${ }^{26}$. 


\begin{abstract}
Methods
PCR based method for variants creation. Variants were created using the QuikChange protocol with Hot Start DNA polymerase from Thermococcus kodakaraensis. An aliquot of $50 \mu \mathrm{l}$ reaction mixtures typically contained $30 \mu \mathrm{l}$ water, $5 \mu \mathrm{l}$ DNA polymerase buffer $(\times 10), 3 \mu \mathrm{l} 25 \mathrm{mM} \mathrm{MgSO}_{4}, 5 \mu \mathrm{l} 2 \mathrm{mM}$ dNTPs, $2.5 \mu \mathrm{l}$ DMSO, $0.5 \mu \mathrm{l}(50-100 \mathrm{ng})$ template DNA, $0.5 \mu \mathrm{l}$ for each primer $(100 \mu \mathrm{M})$ and $1 \mu \mathrm{l} \mathrm{KOD}$ hot start polymerase. The PCR conditions are as follows: $95^{\circ} \mathrm{C} 3 \mathrm{~min}$, $\left(95^{\circ} \mathrm{C} 30 \mathrm{~s}, 60^{\circ} \mathrm{C} 30 \mathrm{~s}, 68^{\circ} \mathrm{C} 5 \mathrm{~min} 30 \mathrm{~s}\right) \times 24$ cycles, $68^{\circ} \mathrm{C} 10 \mathrm{~min}, 16^{\circ} \mathrm{C} 30 \mathrm{~min}$ The PCR products were analysed on agarose gel by electrophoresis and purified using a Qiagen PCR gel extraction kit. A total of $2 \mu \mathrm{l}$ NEB CutSmart Buffer and $2 \mu \mathrm{l}$ $D p n$ I were added in $50 \mu \mathrm{l}$ PCR reaction mixture and the digestion was carried out at $37^{\circ} \mathrm{C}$ for $6 \mathrm{~h}$. After $D p n$ I digestion, the PCR products $(1 \mu \mathrm{l})$ were directly transformed into electro-competent Escherichia coli BL21(DE3) to create the variants for screening. All primers used are listed in Supplementary Table 4.
\end{abstract}

Protein production and purification. The variants selected were produced and purified for biochemical characterization. E. coli BL21(DE3) cells were transformed with the plasmids prsfDuet-1 (Novagen) ${ }^{38}$ containing the gene of interest. To ensure monoclonality, single-colony streakouts were first prepared and inoculated into $4 \mathrm{ml} \mathrm{LB}$ medium containing $50 \mu \mathrm{g} \mathrm{ml}^{-1}$ kanamycin and cultured overnight at $37^{\circ} \mathrm{C}, 220$ r.p.m. The overnight culture $(4 \mathrm{ml})$ was transferred into $200 \mathrm{ml}$ TB with $50 \mu \mathrm{g} \mathrm{ml}^{-1}$ kanamycin in $500 \mathrm{ml}$ shaking flasks. The cultivation continued at $37^{\circ} \mathrm{C}, 220$ r.p.m. for $2-3 \mathrm{~h}$ until the $\mathrm{OD}_{600}$ reached $0.6-0.8$, then IPTG was added to a final concentration of $0.2 \mathrm{mM}$ and the temperature was reduced to $25^{\circ} \mathrm{C}$. After $20 \mathrm{~h}$ of expression, the cells were collected by centrifugation at 4,000 r.p.m., $4^{\circ} \mathrm{C}$ for $15 \mathrm{~min}$. The cell pellets were stored at $-80^{\circ} \mathrm{C}$ until further processing. The cell pellets were disrupted by sonication and the tube was kept in an ice bath during sonication. The collected lysate was centrifuged for $45 \mathrm{~min}$ at 11,000 r.p.m. at $4{ }^{\circ} \mathrm{C}$ and the obtained brownish-red supernatant was filtered to sterility with a $0.45 \mu \mathrm{m}$ filter. The lysate obtained was loaded onto a nickel affinity column (GE Healthcare) and washed with $10-250 \mathrm{mM}$ imidazole solution containing $800 \mathrm{mM} \mathrm{NaCl}$ and $50 \mathrm{mM}$ potassium phosphate buffer $(\mathrm{pH} 8.0)$. Proteins from the flow through were pooled and concentrated, and then desalted using Hitrap desalting column equilibrated with $100 \mathrm{mM}$ potassium phosphate buffer ( $\mathrm{pH} \mathrm{8.0)}$ ). A flow rate of $5 \mathrm{ml} \mathrm{min}-1$ was used and all fractions showing adsorption at $417 \mathrm{~nm}$ were collected and concentrated to a final volume of $1 \mathrm{ml}$ with Amicon Ultra centrifugal filters (cutoff $50 \mathrm{kDa}$ ). The protein was shock frozen with liquid nitrogen and stored at $-80^{\circ} \mathrm{C}$ until further usage (see Supplementary Fig. 13 for protein SDS-PAGE analysis).

Determination of enzyme concentration. Total enzyme concentration including the active P450 and inactive forms P420 (no monooxygenation activity) was determined by $\mathrm{CO}$ difference spectrum analysis ${ }^{39}$ for both purified enzyme and CFE. The extinction coefficients of $\varepsilon_{450-490}=91,000 \mathrm{M}^{-1} \mathrm{~cm}^{-1}$ and $\varepsilon_{420-490}=110,000 \mathrm{M}^{-1} \mathrm{~cm}^{-1}$ were used for the P450 and cytochrome P420, respectively. For the serine ligated mutants $\mathrm{P} 411$, an extinction coefficient of $\varepsilon_{411-490}=103,000 \mathrm{M}^{-1} \mathrm{~cm}^{-1}$ was employed ${ }^{40}$. Kemp elimination activity was measured based on the total enzyme concentration since the enzyme without monooxygenation activity is still active towards the Kemp substrate.

Substrate solubility. Solubility of 5-nitrobenzisoxazole (substrate 1) under the assay conditions $(50 \mathrm{mM}$ sodium phosphate buffer with $100 \mathrm{mM} \mathrm{NaCl}, \mathrm{pH} 8.0,5 \%$ acetonitrile $\left.(\mathrm{ACN}), 25^{\circ} \mathrm{C}\right)$ was quantified according to the previously reported method $^{16}$. The solubility limit of 5 -nitrobenzisoxazole under these conditions was determined to be $3.2 \mathrm{mM}$.

Kinetic assay. The assays were conducted on a JASCO V-650 spectrophotometer with quartz cuvettes monitoring absorbance at $380 \mathrm{~nm}$ at $25^{\circ} \mathrm{C}$ using at least three independent measurements. In a typical experiment the purified enzyme (with final concentration ranging from 0.1 to $0.3 \mu \mathrm{M}$ ) was added to freshly prepared 5 -nitrobenzisoxazole substrate ( $50 \mu \mathrm{M}-2 \mathrm{mM}$ final concentration) in $50 \mathrm{mM}$ sodium phosphate buffer ( $\mathrm{pH} 8.0$ ) containing $100 \mathrm{mM} \mathrm{NaCl}$ and $5 \% \mathrm{ACN}$. The reactions were initiated by adding NADPH with a final concentration of $0.25 \mathrm{mM}$. The slope before addition of NADPH was subtracted as background. Product's extinction coefficient $\left(15,800 \mathrm{~cm}^{-1} \mathrm{M}^{-1}\right)$ was used ${ }^{8}$. Initial rates divided by catalyst concentration were plotted against substrate concentration, and $k_{\text {cat }}$ and $K_{\mathrm{m}}$ values were determined by fitting the data to the Michaelis-Menten equation $v_{0} /[E]_{0}=k_{\text {cat }}[S] /\left(K_{\mathrm{m}}+[S]\right)$. In the case of WT P450-BM3 and variant-F87Icatalysed cleavage of 5-nitrobenzisoxazole, the individual of $k_{\text {cat }}$ and $K_{\mathrm{m}}$ values could not be determined due to limited substrate solubility, only the enzymatic efficiency $\left(k_{\mathrm{cat}} / K_{\mathrm{m}}\right)$ could be measured by fitting the linear portion of the Michaelis-Menten graph to the following equation: $v_{0} /[E]_{0}=\left(k_{\text {cat }} / K_{\mathrm{m}}\right)[S]$, the $K_{\mathrm{m}}$ and $k_{\text {cat }}$ values were estimated based on the linearity of the graph. The kinetic data is presented in Table 1 and Supplementary Fig. 14.

Activity assay under anaerobic conditions. Stock solutions of substrate in acetonitrile, NADPH and P450-BM3 in phosphate buffer (50 mM, pH 8.0, $100 \mathrm{mM}$ $\mathrm{NaCl}$ ) were degassed separately by removing the air and flushing argon into solution. In a typical experiment, into a $1 \mathrm{ml}$ spectrophotometer quartz cuvette with a sealing lid (that was previously prepared for anaerobic conditions by removing the air and adding argon using vacuum line), was sequentially added purified protein $(945 \mu \mathrm{l}$ with concentration of WT $1 \mu \mathrm{M})$, substrate $(50 \mu \mathrm{l}$ in ACN, final concentration $0.5 \mathrm{mM}$ ) and then NADPH $(5 \mu \mathrm{l}$, final concentration $0.25 \mathrm{mM}$ ). All three components were carefully added using Hamilton syringes in the cuvette that was continuously flushed with argon via a needle. The cuvette was sealed and immediately subjected to activity assay. To investigate the effect of $\mathrm{CO}$ on activity, the enzyme solution saturated with CO was employed. All assays were performed at least in three independent measurements.

Preparation of reconstituted P450-BM3 protein. The directed expression, Ni-NTA purification, and metalation of apo-P450-BM3 protein to generate the reconstituted $\mathrm{P} 450-\mathrm{BM} 3$ proteins containing haem-Fe (hemin) or haem-Zn(II) were conducted by strictly following the well-tested and reliable procedure ${ }^{29}$. The protein concentration was measured using the Bradford assay. Ultraviolet-vis spectra of reconstituted proteins were tested using a JASCO V-650 spectrophotometer.

MD simulations. The initial structures of P450-BM3 were taken from Protein Data Bank with PDB code of 1JPZ (ref. 28). The substrate 5-nitrobenzisoxazole wa docked into the active site of P450-BM3 using AutoDock Vina tool ${ }^{41}$ in Chimera ${ }^{42}$. The force field for the haem moiety in the resting state (Fe(III)) was taken from the literature ${ }^{43}$, while the force field for the one-electron reduced state ( $\left.\mathrm{Fe}(\mathrm{II})\right)$ was parameterized using 'MCPB.py' model ${ }^{44}$. The general AMBER force field ${ }^{45}$ was used for the substrate 5-nitrobenzisoxazole, while the partial atomic charges and missing parameters for the substrate were obtained from the RESP model ${ }^{46}$, using $\mathrm{HF} / 6-31 \mathrm{G}^{*}$ level of theory. After proper minimizations and equilibrations, a productive MD run of $100 \mathrm{~ns}$ was performed for each system. The Amber ff14SB force field ${ }^{47}$ was employed for the protein in all of the MD simulations. All MD simulations were performed with GPU version of Amber 14 package $^{48}$ (see Supplementary Methods for computational system preparation and setup; as well as other computational details for MD simulations).

QM/MM calculations. Equilibrated snapshots from the MD simulations were taken for the subsequent QM/MM calculations. All QM/MM calculations were performed using ChemShell ${ }^{49,50}$, combining Turbomole ${ }^{51}$ for the QM part and DL_POLY ${ }^{52}$ for the MM part. The MM region was treated by CHARMM27 force field ${ }^{53}$, while $\mathrm{QM}$ region with 58 atoms was treated by the hybrid UB3LYP functional ${ }^{54}$ with two basis sets. For geometry optimization and frequency calculations the all electron basis set of def2-SVP ${ }^{55}$, referred to as B1, was used. The energies are further corrected with the large all-electron basis-set Def2-TZVP (ref. 55), labelled as B2. The empirical dispersion energy correction was calculated for all species by using the DFT-D3 programme ${ }^{56}$ (see Supplementary Methods for more computational details of QM/MM calculations). For comparison, a bigger $\mathrm{QM}$ region with 73 atoms was also tested for the first reaction step of Kemp elimination of $\mathbf{1}$, and the result is not much changed (Supplementary Fig. 15).

Metabolism of leflunomide with WT P450-BM3 and variants. Reaction mixture of $500 \mu \mathrm{l}$ typically contained $1 \mu \mathrm{M}$ enzyme, $500 \mu \mathrm{M}$ leflunomide, $2 \%(\mathrm{v} / \mathrm{v})$ methanol as co-solvent and $1 \mathrm{mM}$ NADPH in phosphate buffer $(50 \mathrm{mM}, \mathrm{pH} 8.0$, $100 \mathrm{mM} \mathrm{NaCl}$ ). The reaction was incubated at $25^{\circ} \mathrm{C}, 1,000$ r.p.m. for $1 \mathrm{~h}$. After reaction, ACN $500 \mu \mathrm{l}$ was added to quench the reaction. The supernatant was obtained by centrifugation at 12,000 r.p.m. for $5 \mathrm{~min}$, filtered and then subjected to HPLC analysis.

HPLC analysis. The quantitative assessments of lefunomide and its Kemp product teriflunomide (A771726) were analysed on a Shimadzu LC-2010CHT system with a column of Zorbax Eclipse 5 XDB-C18 $250 \times 4.6 \mathrm{~mm}$ (Agilent Technologies, Palo Alto, CA) at $40^{\circ} \mathrm{C}$ and $254 \mathrm{~nm}$. A binary gradient consisting of a mixture of $10 \mathrm{mM}$ ammonium formate, $0.1 \%$ formic acid (solvent $\mathrm{A}$ ) and $\mathrm{ACN}$ (solvent $\mathrm{B}$ ) at a flow rate of $1 \mathrm{ml} \mathrm{min}^{-1}$ was employed. The LC gradient was programmed as follows: solvent A to solvent B ratio was held at 100:0 (v/v) for $3 \mathrm{~min}$ and then adjusted from 100:0 (v/v) to 10:90 $(\mathrm{v} / \mathrm{v})$ for 20 min and from 10:90 $(\mathrm{v} / \mathrm{v})$ to 100:0 $(\mathrm{v} / \mathrm{v})$ from 20 to $25 \mathrm{~min}$. Leflunomide and teriflunomide were separated with a retention time of 15.3 and $20.4 \mathrm{~min}$, respectively.

Chemistry. The details can be found in Supplementary Information (see Supplementary Figs 16 and 17; Supplementary Methods for chemicals and GC-MS analysis).

Data availability. The crystal structure used to perform the MD simulation studies is archived from RCSB Protein Data Bank with PDB code of 1JPZ. All other data supporting the findings of this study are available within the paper and its Supplementary Information Files. 


\section{References}

1. Kemp, D., Cox, D. D. \& Paul, K. G. Physical organic chemistry of benzisoxazoles. IV. Origins and catalytic nature of the solvent rate acceleration for the decarboxylation of 3-carboxybenzisoxazoles. J. Am. Chem. Soc. 97, 7312-7318 (1975)

2. Albanese, D. C. \& Gaggero, N. Albumin as a promiscuous biocatalyst in organic synthesis. RSC Adv. 5, 10588-10598 (2015).

3. Thorn, S. N., Daniels, R. G., Auditor, M.-T. M. \& Hilvert, D. Large rate accelerations in antibody catalysis by strategic use of haptenic charge. Nature 373, 228-230 (1995).

4. Hollfelder, F., Kirby, A. J. \& Tawfik, D. S. Off-the-shelf proteins that rival tailor-made antibodies as catalysts. Nature 383, 60-63 (1996).

5. Kikuchi, K., Thorn, S. N. \& Hilvert, D. Albumin-catalyzed proton transfer J. Am. Chem. Soc. 118, 8184-8185 (1996).

6. Boucher, G. et al. Serum albumin-catalyzed trigger system by using a tandem Kemp elimination/ $\beta$-elimination reaction. ChemBioChem. 6, 807-810 (2005).

7. Frushicheva, M. P., Cao, J. \& Warshel, A. Challenges and advances in validating enzyme design proposals: the case of Kemp eliminase catalysis. Biochemistry 50, 3849-3858 (2011).

8. Korendovych, I. V. et al. Design of a switchable eliminase. Proc. Natl Acad. Sci. USA 108, 6823-6827 (2011).

9. Moroz, O. V. et al. A single mutation in a regulatory protein produces evolvable allosterically regulated catalyst of nonnatural reaction. Angew. Chem. Int. Ed. 125, 6366-6369 (2013).

10. Röthlisberger, D. et al. Kemp elimination catalysts by computational enzyme design. Nature 453, 190-195 (2008).

11. Khersonsky, O. et al. Evolutionary optimization of computationally designed enzymes: Kemp eliminases of the KE07 series. J. Mol. Biol. 396, 1025-1042 (2010).

12. Merski, M. \& Shoichet, B. K. Engineering a model protein cavity to catalyze the Kemp elimination. Proc. Natl Acad. Sci. USA 109, 16179-16183 (2012).

13. Khersonsky, O. et al. Bridging the gaps in design methodologies by evolutionary optimization of the stability and proficiency of designed Kemp eliminase KE59. Proc. Natl Acad. Sci. USA 109, 10358-10363 (2012)

14. Moroz, Y. S. et al. New tricks for old proteins: single mutations in a nonenzymatic protein give rise to various enzymatic activities. J. Am. Chem. Soc. 137, 14905-14911 (2015).

15. Privett, H. K. et al. Iterative approach to computational enzyme design. Proc. Natl Acad. Sci. USA 109, 3790-3795 (2012).

16. Blomberg, R. et al. Precision is essential for efficient catalysis in an evolved Kemp eliminase. Nature 503, 418-421 (2013).

17. Korendovych, I. V. \& DeGrado, W. F. Catalytic efficiency of designed catalytic proteins. Curr. Opin. Struct. Biol. 27, 113-121 (2014).

18. Khersonsky, O., Malitsky, S., Rogachev, I. \& Tawfik, D. S. Role of chemistry versus substrate binding in recruiting promiscuous enzyme functions. Biochemistry 50, 2683-2690 (2011).

19. Isin, E. M. \& Guengerich, F. P. Complex reactions catalyzed by cytochrome P450 enzymes. Biochim. Biophys. Acta 1770, 314-329 (2007).

20. Guengerich, F. P. Fifty Years of Cytochrome P450 Research 17-41 (Springer, 2014).

21. Ortiz de Montellano, P. R. Hydrocarbon hydroxylation by Cytochrome 450 enzymes. Chem. Rev. 110, 932-948 (2010).

22. Kalgutkar, A. S. et al. In vitro metabolism studies on the isoxazole ring scission in the anti-inflammatory agent leflunomide to its active $\alpha$-cyanoenol metabolite A771726: mechanistic similarities with the cytochrome P450-catalyzed dehydration of aldoximes. Drug Metab. Dispos. 31, 1240-1250 (2003).

23. Yu, J. et al. Elucidation of a novel bioactivation pathway of a 3, 4-unsubstituted isoxazole in human liver microsomes: formation of a glutathione adduct of a cyanoacrolein derivative after isoxazole ring opening. Drug Metab. Dispos. 39, 302-311 (2011)

24. O'Donnell, E. F. et al. The anti-inflammatory drug leflunomide is an agonist of the aryl hydrocarbon receptor. PLoS ONE 5, e13128 (2010).

25. Svastits, E. W., Dawson, J. H., Breslow, R. \& Gellman, S. H. Functionalized nitrogen atom transfer catalyzed by cytochrome P-450. J. Am. Chem. Soc. 107, 6427-6428 (1985)

26. Renata, H., Wang, Z. J. \& Arnold, F. H. Expanding the enzyme universe: accessing non-natural reactions by mechanism-guided directed evolution. Angew. Chem. Int. Ed. 54, 3351-3367 (2015).

27. Boucher, J.-L., Delaforge, M. \& Mansuy, D. Dehydration of alkyl-and arylaldoximes as a new cytochrome P450-catalyzed reaction: mechanism and stereochemical characteristics. Biochemistry 33, 7811-7818 (1994).

28. Haines, D. C., Tomchick, D. R., Machius, M. \& Peterson, J. A. Pivotal role of water in the mechanism of P450BM-3. Biochemistry 40, 13456-13465 (2001).

29. Key, H. M., Dydio, P., Clark, D. S. \& Hartwig, J. F. Abiological catalysis by artificial haem proteins containing noble metals in place of iron. Nature 534, 534-537 (2016)

30. Perera, R., Sono, M. \& Dawson, J. H. Molecular basis for the inability of an oxygen atom donor ligand to replace the natural sulfur donor heme axial ligand in cytochrome 450 catalysis. Spectroscopic characterization of the Cys436Ser CYP2B4 mutant. Arch. Biochem. Biophys. 507, 119-125 (2011).

31. Shaik, S. et al. P450 enzymes: their structure, reactivity, and selectivity-modeled by QM/MM calculations. Chem. Rev. 110, 949-1017 (2010).

32. Schyman, P., Lai, W., Chen, H., Wang, Y. \& Shaik, S. The directive of the protein: how does cytochrome $\mathrm{P} 450$ select the mechanism of dopamine formation? J. Am. Chem. Soc. 133, 7977-7984 (2011).

33. Dubey, K. D., Wang, B. \& Shaik, S. Molecular dynamics and QM/MM calculations predict the substrate-induced gating of cytochrome P450 BM3 and the regio and stereo-selctivity of fatty acid hydroxylation. J. Am. Chem. Soc. 138, 837-845 (2016).

34. Shaik, S., Filatov, M., Schröder, D. \& Schwarz, H. Electronic structure makes a difference: cytochrome P-450 mediated hydroxylations of hydrocarbons as a two-state reactivity paradigm. Chem. Eur. J. 4, 193-199 (1998).

35. Schröder, D., Shaik, S. \& Schwarz, H. Two-state reactivity as a new concept in organometallic chemistry. Accounts Chem. Res. 33, 139-145 (2000)

36. Huang, W.-C. et al. Filling a hole in cytochrome P450 BM3 improves substrate binding and catalytic efficiency. J. Mol. Biol. 373, 633-651 (2007).

37. Chan, E. C., New, L. S., Yap, C. W. \& Goh, L. T. Pharmaceutical metabolite profiling using quadrupole/ion mobility spectrometry/time-of-flight mass spectrometry. Rapid Commun. Mass Spectrom. 23, 384-394 (2009).

38. Agudo, R., Roiban, G.-D., Lonsdale, R., Ilie, A. \& Reetz, M. T. Biocatalytic route to chiral acyloins: P450-catalyzed regio-and enantioselective $\alpha$-hydroxylation of ketones. J. Org. Chem. 80, 950-956 (2014).

39. Guengerich, F. P., Martin, M. V., Sohl, C. D. \& Cheng, Q. Measurement of cytochrome P450 and NADPH-cytochrome P450 reductase. Nat. Protoc. 4, 1245-1251 (2009).

40. Coelho, P. S. et al. A serine-substituted P450 catalyzes highly efficient carbene transfer to olefins in vivo. Nat. Chem. Biol. 9, 485-487 (2013).

41. Trott, O. \& Olson, A. J. AutoDock Vina: improving the speed and accuracy of docking with a new scoring function, efficient optimization, and multithreading. J Comput. Chem. 31, 455-461 (2010).

42. Pettersen, E. F. et al. UCSF Chimera-a visualization system for exploratory research and analysis. J. Comput. Chem. 25, 1605-1612 (2004).

43. Shahrokh, K., Orendt, A., Yost, G. S. \& Cheatham, T. E. Quantum mechanically derived AMBER-compatible heme parameters for various states of the cytochrome P450 catalytic cycle. J. Comput. Chem. 33, 119-133 (2012).

44. Li, P. \& Merz, Jr K. M. MCPB.py: a python based metal center parameter builder. J. Chem. Inf. Model. 56, 599-604 (2016).

45. Wang, J., Wolf, R. M., Caldwell, J. W., Kollman, P. A. \& Case, D. A. Development and testing of a general amber force field. J. Comput. Chem. 25, 1157-1174 (2004).

46. Bayly, C. I., Cieplak, P., Cornell, W. D. \& Kollman, P. A. A Well-behaved electrostatic potential based method using charge restraints for deriving atomic charges: the RESP model. J. Phys. Chem. 97, 10269-10280 (1993).

47. Maier, J. A. et al. ff14sb: Improving the accuracy of protein side chain and backbone parameters from ff99sb. J. Chem. Theory Comput. 11, 696-3713 (2015).

48. Salomon-Ferrer, R., Götz, A. W., Poole, D., Le Grand, S. \& Walker, R. C. Routine microsecond molecular dynamics simulations with AMBER on GPUs. 2. Explicit solvent particle mesh Ewald. J. Chem. Theory Comput. 9, 3878-3888 (2013).

49. Sherwood, P. et al. QUASI: a general purpose implementation of the QM/MM approach and its application to problems in catalysis. J. Mol. Struct. 632, 1-28 (2003).

50. Metz, S., Kästner, J., Sokol, A. A., Keal, T. W. \& Sherwood, P. ChemShell-a modular software package for QM/MM simulations. Comput. Mol. Sci. 4, 101-110 (2014).

51. Ahlrichs, R., Bär, M., Häser, M., Horn, H. \& Kölmel, C. Electronic structure calculations on workstation computers: the program system turbomole. Chem. Phys. Lett. 162, 165-169 (1989).

52. Smith, W. \& Forester, T. DL_POLY_2. 0: a general-purpose parallel molecular dynamics simulation package. J. Mol. Graph. 14, 136-141 (1996).

53. Brooks, B. R. et al. CHARMM: the biomolecular simulation program. J. Comput. Chem. 30, 1545-1614 (2009).

54. Lee, C. T., Yang, W. T. \& Parr, R. G. Development of the Colle-Salvetti correlation-energy formula into a functional of the electrondensity. Phys. Rev. B 37, 785-789 (1988).

55. Weigend, F. \& Ahlrichs, R. Balanced basis sets of split valence, triple zeta valence and quadruple zeta valence quality for $\mathrm{H}$ to $\mathrm{Rn}$ : design and assessment of accuracy. Phys. Chem. Chem. Phys. 7, 3297-3305 (2005).

56. Grimme, S., Antony, J., Ehrlich, S. \& Krieg, H. A consistent and accurate ab initio parametrization of density functional dispersion correction (DFT-D) for the 94 elements H-Pu. J. Chem. Phys. 132, 154104-1-154104-19 (2010).

\section{Acknowledgements}

M.T.R. thanks the Max-Planck-Society (reference number 106705) and the LOEWE Research cluster SynChemBio (reference number 56150029) for generous support. 
S.S. acknowledges support from the Israel Science Foundation (ISF grant 1183/13), and B.W. and K.D.D. are supported in part by a PBC fellowship. G.B. thanks the LOEWE initiative of the state of Hessen for support, and I.V.K. acknowledges support from the Alexander von Humboldt Foundation (reference number 61508772) and the NIH (grant GM 119634).

\section{Author contributions}

A.L. and A.I. performed the experiments; B.W. and K.D.D. performed the MD simulations and QM/MM study; the project is supervised by M.T.R. and S.S.; A.L., B.W., S.S.,

G.B., I.V.K. and M.T.R. wrote the manuscript; all authors checked the manuscript.

\section{Additional information}

Supplementary Information accompanies this paper at http://www.nature.com/ naturecommunications

Competing interests: The authors declare no competing financial interests.
Reprints and permission information is available online at http://npg.nature.com/ reprintsandpermissions/

How to cite this article: $\mathrm{Li}$, A. et al. A redox-mediated Kemp eliminase. Nat. Commun 8, 14876 doi: $10.1038 /$ ncomms14876 (2017).

Publisher's note: Springer Nature remains neutral with regard to jurisdictional claims in published maps and institutional affiliations.

\section{(c) (1)}

This work is licensed under a Creative Commons Attribution 4.0 International License. The images or other third party material in this article are included in the article's Creative Commons license, unless indicated otherwise in the credit line; if the material is not included under the Creative Commons license, users will need to obtain permission from the license holder to reproduce the material. To view a copy of this license, visit http://creativecommons.org/licenses/by/4.0/

(C) The Author(s) 2017 